\title{
Diversification, performance and optimal business mix of insurance portfolios
}

\author{
Hyun Tae Kim $^{1}$ \\ ${ }^{1}$ Department of Applied Statistics, Yonsei University \\ Received 4 October 2013, revised 21 October 2013, accepted 28 October 2013
}

\begin{abstract}
For multi-line insurance companies, allocating the risk capital to each line is a widelyaccepted risk management exercise. In this article we consider several applications of the Euler capital allocation. First, we propose visual tools to present the diversification and the line-wise performance for a given loss portfolio so that the risk managers can understand the interactions among the lines. Secondly, on theoretical side, we prove that the Euler allocation is the directional derivative of the marginal or incremental allocation method, an alternative capital allocation rule in the literature. Lastly, we establish the equivalence between the mean-shortfall optimization and the RORAC optimization when the risk adjusted capital is the expected shortfall, and show how to construct the optimal insurance business mix that maximizes the portfolio RORAC. An actual loss sample of an insurance portfolio is used for numerical illustrations.
\end{abstract}

Keywords: Capital allocation, Euler allocation, mean-shortfall optimization, risk measure, RORAC optimization.

\section{Introduction and motivation}

For financial firms with various business lines, allocating the firm-level solvency risk capital back to individual lines is a widely-accepted risk management exercise, eg, Dev (2004), McNeil et al. (2005) and Tasche (2004). Consider the aggregate loss of such a firm with $d$ different lines for a single period:

$$
S=X_{1}+X_{2}+\ldots+X_{d}
$$

where $X_{i}$ represents the net loss random variable (rv) of the $i$ th line. The standard theory states that the risk capital of the entire firm is written as $\rho(S)$, where $\rho$ is a suitably chosen risk measure with the Value-at-Risk (VaR) and the Expected Shortfall (ES in short; also known as Tail VaR, Conditional VaR or Conditional Tail Expectation) being the prominent examples.

Once the entire firm's risk capital has been set, it can be allocated to each line of business to measure the line-wise risk contribution and performance. Mathematically a capital allocation rule is a mapping from the aggregate loss rv $S$ in (1.1) to a vector of $d$ dimension $\left(A C_{1}, \ldots, A C_{d}\right)$, such that

$$
\sum_{i=1}^{d} A C_{i}=\rho(S) .
$$

Here $A C_{i}$ represents the allocated capital for line $i$, taking a specific form under the given allocation rule. A good capital allocation rule is useful in assessing and comparing the performance

\footnotetext{
1 Assistant professor, Department of Applied Statistics, Yonsei University, Seoul 120-749, Korea.

Email: jhtkim@yonsei.ac.kr
} 
of each line, in product pricing and in strategic planning for different business blocks within a multi-line company.

Finding sensible capital allocation rules and their economic or mathematical justifications has produced much discussion recently, see, eg, Denault (2001), Myers and Read Jr. (2001), Kalkbrener (2005), Kim and Hardy (2009) and Dhaene et al. (2012). Other authors have investigated capital allocation from a (financial) economic perspective, where motivations are based on corporate finance theory, such as limited liability, frictional costs and market efficiency, eg, Myers and Read Jr. (2001), Merton and Perold (1993), Zanjani (2002) and Sherris (2006), to name a few. Another class of capital allocation, called the marginal allocation method, has been used in the literature; eg, Matten (1996), Chew (2001), Albrecht (2006). The idea of the marginal allocation is based on the incremental increase in the risk capital due to the addition of the corresponding line of business. Among others, the Euler principle by Tasche (1999, 2004, 2007), Hallerbach (2003) and Gourieroux et al. (2000) is a notable method that provides an economic basis for measuring line-wise performance using the return on risk adjusted capital. Related to this, the notion of the diversification index for a portfolio under the Euler allocation is introduced and discussed in, e.g., Tasche (2004, 2006, 2007), Memmel and Wehn (2006) and Garcia Cespedes et al. (2006), for a given risk measure, Skoglund and Chen (2009) developed an information measure, based on the Kullback information theory, that conveys similar information to the Euler decomposition for non-linear portfolios.

The present article considers several practical (1 below) and theoretical (2 and 3) applications of the Euler allocation problem in the insurance portfolio context, with the common keywords being the diversification index (DI) and the return on risk adjusted capital (RORAC), the two articulated risk metrics of Tasche $(1999,2006,2007)$. The main contributions and motivations of this article are as follows:

1. Several graphical tools to visualize the DI and the RORAC are proposed in order to fill the gap between the current theory and the mundane use of the relevant graphs in practice. For instance, Figure 1.1, being a typical presentation of a capital allocation result for a multi-line firm, shows risk-return information for each line, but fails to contain the valuable information on the interactions among the lines; the numbers will be discussed in the next section. The proposed graphs, based on the random permutation, extends such simple figures so that proper information on the DI and the RORAC can be efficiently captured.

2. While the Euler allocation is a popular method to allocate capitals, there have been other proposals in the literature. We show that there is a close relationship between the Euler allocation and the marginal allocation. In particular, we prove that the former is the directional derivative of the latter.

3. A crucial question raised by management is to find the optimal business mix to maximize the RORAC of the firm. RORAC optimization has been carried out through trial and error in previous research. We show that the optimal business mix can be obtained by adapting the mean-ES optimization technique (Rockafellar and Uryasev, 2000; Bertsimas et al., 2004) when the risk measure is the ES. Once optimized, the proposed visual graph successfully captures the optimal state of the portfolio.

For illustration and optimization throughout this article, we use the Euler allocation of the ES in (2.3), because the ES is coherent (Artzner et al., 1999) and preferred in many applications; see also Shim and Hwang (2013) for estimating the ES using kernel machines.

This article is organized as follows. In Section 2, some background is given along with the dataset to be used throughout this article. Section 3 proposes two graphs for visualizing the diversification index and the performance of individual lines. We discuss the relationship between 


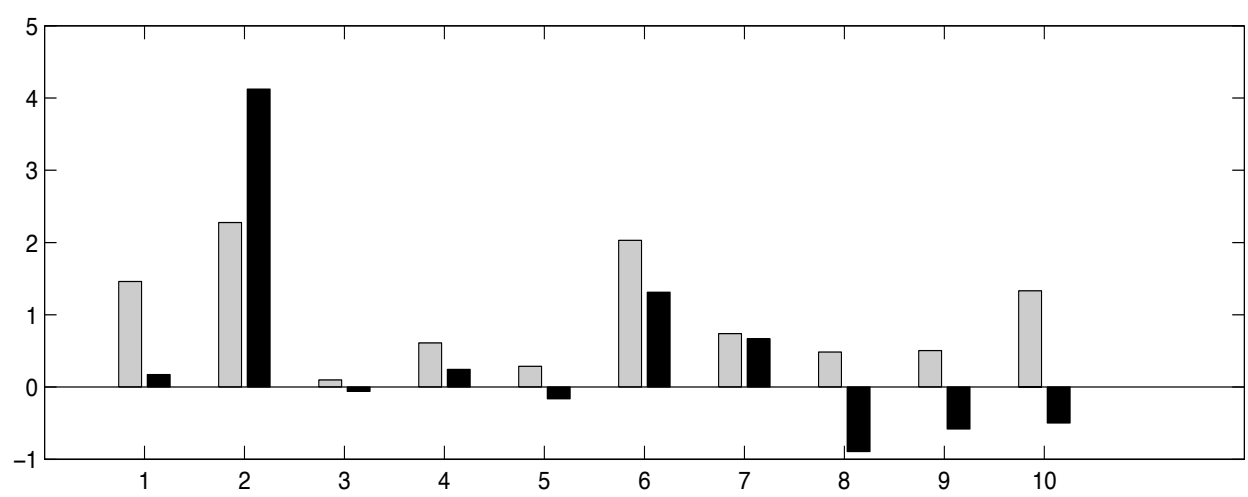

Figure 1.1 Profit (grey) and allocated capital (black) of ten different lines of a firm

the Euler and the marginal allocation rules in Section 4. The RORAC optimization and its result on the working dataset are presented in Section 5. Section 6 provides brief concluding remarks.

\section{Preliminaries}

\subsection{Euler allocation}

The Euler principle of capital allocation has been discussed in Tasche (2004, 2006, 2007), Memmel and Wehn (2006) and Garcia Cespedes et al. (2006). Let us denote the portfolio of risks under consideration by $\Omega=\left\{X_{1}, \ldots, X_{d}\right\}$ with $S=\sum_{1}^{d} X_{i}$ as before. When the firm's risk capital is given by a risk measure $\rho(S)$, where $\rho$ is positive homogeneous (that is, $\rho(t X)=t \rho(X)$ for a constant $t$ ) and continuously differentiable, its Euler allocation is defined as the directional derivative with respect to each line:

$$
\rho\left(X_{i} \mid \Omega\right)=\left.\frac{d \rho}{d h}\left(S+h X_{i}\right)\right|_{h=0} .
$$

When the risk measure is the ES of a continuous loss $\mathrm{rv} S$, for a given probability level $p$, defined by

$$
\rho(S)=E S_{p}(S)=E\left[S \mid S>Q_{p}(S)\right], \quad 0<p<1,
$$

where $Q_{p}(S)=F^{-1}(p)$ is the quantile function of $S$ at $p$, the Euler allocated capital of the $i$ th line is given by

$$
\rho\left(X_{i} \mid \Omega\right)=E\left[X_{i} \mid S>Q_{p}(S)\right] .
$$

Other examples in this class under some technical conditions include the covariance allocation

$$
\rho\left(X_{i} \mid \Omega\right)=\frac{\operatorname{Cov}\left(X_{i}, S\right)}{\sqrt{\operatorname{Var}(S)}}
$$

and the VaR allocation

$$
\rho\left(X_{i} \mid \Omega\right)=E\left(X_{i} \mid S=Q_{S}(p)\right) .
$$

The expressions of the ES and its allocated capitals take further specific forms depending on the joint distribution of $\left(X_{1}, \ldots, X_{d}\right)$, but, from (2.3), the allocated capital is generally nonlinear in $X_{i}$ 
due to the dependence within the portfolio. Thus, allocated capitals contain valuable information on diversification benefit of the portfolio.

In practice the chosen risk measure and its allocation are estimated from a loss sample, either internally generated or historically recorded. Suppose there is an independent and identically distributed (iid) loss random sample of size $n, \mathbf{X}_{1}, \mathbf{X}_{2}, \ldots, \mathbf{X}_{n}$, from the joint distribution of $\left(X_{1}, \ldots, X_{d}\right)$ with the $i$ th observation is the vector

$$
\mathbf{X}_{i}=\left[X_{i 1}, X_{i 2}, \ldots, X_{i d}\right], \quad i=1, \ldots, n .
$$

It is often convenient to create a loss matrix based on these $n$ loss vectors:

$$
\mathbf{M}=\left[\begin{array}{cccc}
X_{11} & X_{12} & \ldots & X_{1 d} \\
X_{21} & X_{22} & \ldots & X_{2 d} \\
\vdots & \vdots & \vdots & \vdots \\
X_{n 1} & X_{n 2} & \ldots & X_{n d}
\end{array}\right]
$$

Then, $\rho(S)$ and $\rho\left(X_{i} \mid \Omega\right)$ are empirically estimated from M. We will refer to this matrix later.

\subsection{Working example}

We make extensive use of the actual loss data from Panjer (2002) throughout this article. Sherris and van der Hoek (2006) also use the same data to obtain an allocation of the insurer default option value in a closed form. The data is a loss sample of an actual financial firm with ten business lines generated from a series of internal simulations.

We assume a multivariate normal (MVN) distribution for the net loss as in the original paper, but we emphasize that the normality of the data is not required for the developments of the present article. We keep the identical dependence structure and the amount of loss across the lines of the data, with the exception of each line's premium at our own discretion, as the premium amount reported is too large to produce loss for the aggregate position under the given parameters. The premium for each line is set as

$$
\operatorname{Prem}_{i}=E\left(Y_{i}\right)+0.5 \cdot \sqrt{\operatorname{Var}\left(Y_{i}\right)}
$$

where $Y_{i}$ is the loss rv of Line $i$. With this specification the net loss of the entire firm is given by

$$
S=\sum_{i=1}^{d} X_{i}=\sum_{i=1}^{d}\left(Y_{i}-\text { Prem }_{i}\right) .
$$

Other cash flows, such as administrative and underwriting costs for different lines or investment incomes, are ignored but can easily be adapted by suitable adjustments. From the fitted model we generate a sample of size 1,000. Table (2.1) shows a summary of the sample statistics and the Euler allocation at the risk capital set at ES $95 \%$ of $S$. Line 2 has the largest positive correlation with the aggregate $S$, followed by Lines 6,7 and 4 , and their allocated capitals suggest that these four lines mainly drive the total risk. The negative correlations of Lines 8 and 9 to $S$ indicate their hedging positions. Lines 2 and 6 have the largest variance. A standard summary figure is presented in Figure (1.1), but fails to capture the dependence information, such as risk pooling and diversification. 
Table 2.1 Summary of the net loss generated from Panjer (2002)

\begin{tabular}{cccccccccccc}
\hline \hline Corr & $S$ & 1 & 2 & 3 & 4 & 5 & 6 & 7 & 8 & 9 & 10 \\
\hline$S$ & 1.00 & 0.27 & 0.67 & 0.11 & 0.38 & 0.14 & 0.40 & 0.39 & -0.14 & -0.05 & 0.10 \\
1 &. & 1.00 & 0.01 & 0.17 & 0.02 & 0.21 & -0.24 & -0.14 & 0.14 & 0.07 & -0.08 \\
2 &. &. & 1.00 & 0.06 & 0.32 & -0.03 & 0.02 & 0.19 & -0.18 & -0.12 & -0.22 \\
3 &. &. &. & 1.00 & -0.01 & -0.07 & 0.06 & 0.06 & -0.08 & -0.09 & -0.11 \\
4 &. &. &. &. & 1.00 & 0.22 & 0.04 & 0.08 & -0.11 & 0.12 & -0.26 \\
5 &. &. &. &. &. & 1.00 & -0.13 & -0.00 & 0.00 & 0.15 & -0.00 \\
6 &. &. &. &. &. &. & 1.00 & 0.10 & -0.08 & -0.43 & -0.11 \\
7 &. &. &. &. &. &. &. & 1.00 & -0.23 & 0.05 & 0.07 \\
8 &. &. &. &. &. &. &. &. & 1.00 & -0.15 & -0.17 \\
9 &. &. &. &. &. &. &. &. &. & 1.00 & 0.19 \\
10 &. &. &. &. &. &. &. &. &. &. & 1.00 \\
\hline Mean & -9.82 & -1.46 & -2.28 & -0.10 & -0.61 & -0.29 & -2.03 & -0.74 & -0.48 & -0.50 & -1.33 \\
\hline Std & 6.70 & 2.79 & 4.52 & 0.19 & 1.33 & 0.54 & 3.88 & 1.65 & 0.95 & 1.04 & 2.55 \\
\hline Capital & 4.32 & 0.17 & 4.12 & -0.06 & 0.24 & -0.16 & 1.31 & 0.67 & -0.89 & -0.58 & -0.50 \\
\hline Prem & 143.80 & 27.04 & 40.09 & 0.95 & 13.36 & 0.43 & 25.99 & 15.21 & 4.97 & 4.92 & 10.86 \\
\hline
\end{tabular}

\section{Visualizing the portfolio}

\subsection{Random permutation}

The permutation method is a standard non-parametric method to test hypotheses and obtain confidence intervals for a parameter, but its use has been limited in statistical applications; see, eg, Good (2000). We use the random permutation to create a benchmark for the dependence structure of the existing portfolio. For our purpose, the null hypothesis is

$$
H_{0}: \text { All lines are independent. }
$$

As the dependence structure among lines is contained in the relationships among columns in matrix $\mathbf{M}$ in (2.6), permuting elements randomly within a column vector will distort the underlying dependence. However, if all lines are indeed independent (that is, $H_{0}$ is true), we expect the permutations to have no impact on the ES and its allocation, because the dependence structure will remain the same. Therefore, by comparing the allocated capitals under the current portfolio against those under $H_{0}$, we can measure how far the current dependence is from independence. To compute the Euler allocation under $H_{0}$, the following steps are taken:

1. Set $r=1$.

2. For columns 1 to $d$ of $\mathbf{M}$, randomly rearrange the elements within each column, and name the resulting loss matrix $\mathbf{M}^{* r}$.

3. Compute the risk capital and its allocations from $\mathbf{M}^{* r}$ :

$$
\rho(S)^{* r}, \rho\left(X_{1} \mid \Omega\right)^{* r}, \ldots, \rho\left(X_{d} \mid \Omega\right)^{* r} .
$$

4. Set $r=r+1$ and repeat Steps 2 to 4 .

After repeating thes steps $R$ times, the resulting risk capital and its allocation estimates (3.2) lead to a distribution of possible outcomes under $H_{0}$. We then take the averages of the total and allocated capital amounts from $R$ permutations to represent the risk measure under independence, and denote them by, respectively,

$$
\rho\left(S \mid \Omega^{\perp}\right)=R^{-1} \sum_{r=1}^{R} \rho(S)^{* r} \quad \text { and } \quad \rho\left(X_{i} \mid \Omega^{\perp}\right)=R^{-1} \sum_{r=1}^{R} \rho\left(X_{i} \mid \Omega\right)^{* r}, \quad i=1, \ldots d,
$$

where the perpendicular sign represents independence. 
We may also test whether all the lines are independent by comparing the empirical estimates against the quantile ranges of the distributions of $R$ estimates in (3.2) at some statistical critical level. If the empirical value falls outside the range, one can validate that the lines are not independent at the given level. Applying random permutations to capital allocation to test independence however is an indirect method as capital allocation is a consequence of underlying dependence. Figure (3.1) shows the distributions of estimates (3.2) for the working data under $H_{0}$ with $R=500,000$. The spikes are the empirical counterparts. It is clear that the lines are dependent as the empirical values are well outside a reasonable range for most lines and for the aggregate loss. For example, the empirical aggregate risk capital, which is 4.32 in the top left panel, lies outside the $99 \%$ confidence interval [4.36,6.94]. In the next subsection, we will use the quantities in (3.3) for a graph visualizing the degree of diversification.
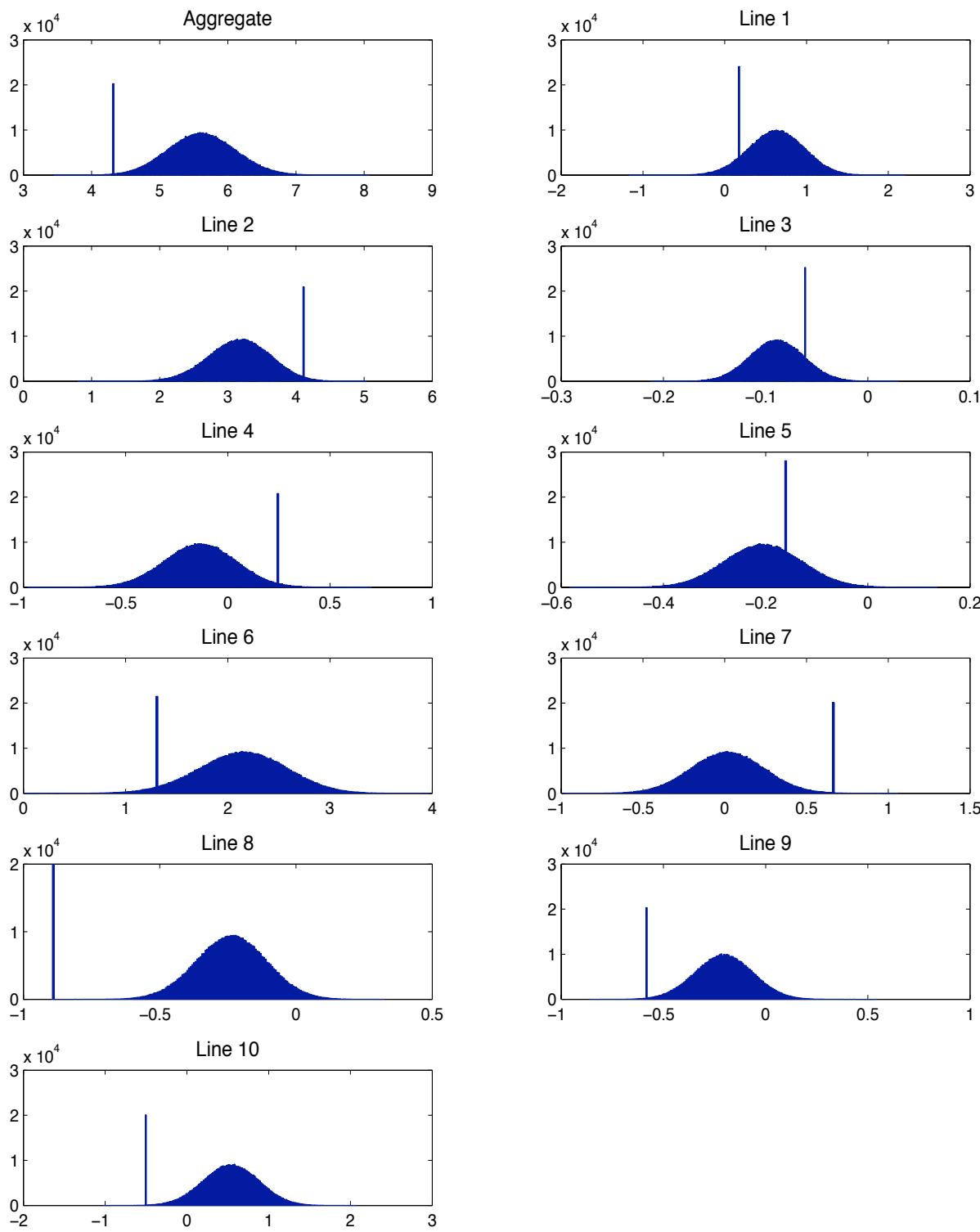

Figure 3.1 SDistribution of the total and allocated capitals under the ES 95\% 
Remark 3.1 For any Line $i$, the largest possible allocation value under permutation is $\rho\left(X_{i}\right)$, which does not need permuting simulation as it is simply the marginal capital. This case represents the worst scenario where all risks are close to comonotonic.

\subsection{Diversification index diagram}

According to the Basel Committee on Banking Supervision (BCBS, 2006, paragraph 770): "A risk concentration is any single exposure or group of exposures with the potential to produce losses large enough (relative to a bank capital, total assets, or overall risk level) to threaten a bank health or ability to maintain its core operations. Risk concentrations are arguably the single most important cause of major problems in banks." In this regard the diversification index (DI) has been introduced and discussed in the literature; Memmel and Wehn (2006), Garcia Cespedes et al. (2006) and more extensively by Tasche $(2004,2006,2007)$. Under the Euler allocation the DI for an entire portfolio and each line is defined by, respectively,

$$
D I(S \mid \Omega)=\frac{\rho(S \mid \Omega)}{\sum_{1}^{d} \rho\left(X_{i}\right)} \quad \text { and } \quad D I\left(X_{i} \mid \Omega\right)=\frac{\rho\left(X_{i} \mid \Omega\right)}{\rho\left(X_{i}\right)} .
$$

We use $\rho(S \mid \Omega)$ instead of $\rho(S)$ to emphasize that the risk capital is computed under the existing dependence. Obviously, the smaller the DI the more diversification benefit the firm has. Tasche (2004) noted the following properties of the DI under the ES:

1. $D I(S \mid \Omega) \leq 1$.

2. $D I(S \mid \Omega) \approx 1$ indicates that $X_{i}$ 's are lmostco-monotonic.

3. $D I\left(X_{i} \mid \Omega\right) \leq 1$.

4. $D I\left(X_{i} \mid \Omega\right)<D I(S \mid \Omega)$ implies that there is some $\epsilon_{i}>0$ such that $D I\left(S+h X_{i} \mid \Omega\right)<D I(S \mid \Omega)$ for $0<h<\epsilon_{i}$.

The last property states that if the $i$ th line's DI is smaller than that of the entire firm, increasing the $i$ th line's volume to some extent guarantees an improvement in the firm's DI. Now we propose a graph termed the DI diagram which visualizes the degree of diversification or concentration of a portfolio using (3.4). In particular, the graph shows the distance between the DI under the existing structure and that under independence. Using the fact that the DI's of the portfolio under independence, from (3.3), are

$$
D I\left(S \mid \Omega^{\perp}\right)=\frac{\rho\left(S \mid \Omega^{\perp}\right)}{\sum_{1}^{d} \rho\left(X_{i}\right)} \quad \text { and } \quad D I\left(X_{i} \mid \Omega^{\perp}\right)=\frac{\rho\left(X_{i} \mid \Omega^{\perp}\right)}{\rho\left(X_{i}\right)},
$$

we mark the position of the $i$ th line on the plane at

$$
\left(x_{i}, y_{i}\right)=\left(D I\left(X_{i} \mid \Omega\right), D I\left(X_{i} \mid \Omega^{\perp}\right)\right)
$$

for $i=1, \ldots, d$, and the entire firm at

$$
\left(x_{S}, y_{S}\right)=\left(D I(S \mid \Omega), D I\left(S \mid \Omega^{\perp}\right)\right) .
$$

Figure (3.2) shows the DI diagram of the working data. The DI diagram essentially compares the DIs for the entire firm and each line under the current dependence against those under independence. As the the capital and its allocation under independence in (3.3) are estimated from the sample and further permutations, it is impossible to pinpoint the permutation that represents exact independence; the corresponding variations can be estimated from (3.2). We find two distinctive usages of the DI diagram: 
Checking independence: If the portfolio indeed had an independent structure, we expect $x_{i}$ to be close to $y_{i}$ so that each $\left(x_{i}, y_{i}\right)$ and $\left(x_{S}, y_{S}\right)$ would lie around the linear function $y=x$. Therefore $y=x$ can be a benchmark of portfolio independence. A line positioned above $y=x$ enjoys less allocated capital than it would under independence. The extra saving - due to the presence of the other lines - puts this line at a strategic advantage as relatively less capital is needed. However, points marked below $y=x$ do not necessarily indicate that they are inferior. It may be the case that these lines takes up more capitals due to the risks transferred from the lines above $y=x$.

Improving diversification: One also can compare $x_{i}$ s with $x_{S}$. According to the fourth property of the DI above, when a line has a lower (better) DI than that of the portfolio, expanding that line by a small amount will reduce the portfolio DI. Therefore, $x=x_{S}$ can be another benchmark line; the lines on the left side of $x=x_{S}$ should be expanded to improve the firm-wide diversification. However the exact size of $\epsilon_{i}$ that guarantees DI improvement is unknown in the literature.

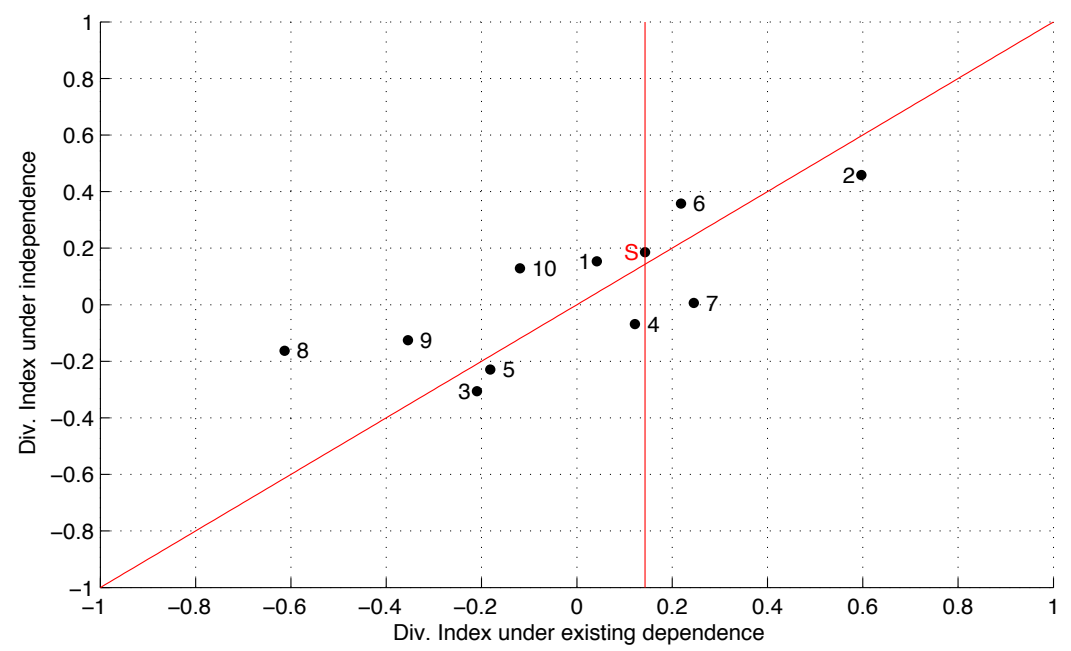

Figure 3.2 DI diagram under ES 95\% allocation with two benchmark lines (entire firm marked as $S$ )

In Figure (3.2), lines 1, 6, 8, 9 and 10 require less capital than they would under independence, indicating their advantage. However as their advantage may arise from the presence of the other lines, one cannot assert the superiority of these lines without referencing the others; for instance, within another firm, these lines may receive larger allocated capitals and would no longer be superior. The aggregate point $S$, marked slightly above $y=x$, indicates that the firm is well balanced, benefiting from pooling risks. If we compare $x_{i}$ s with $x_{S}$, lines 2,6 and 7 need to downsize to improve the portfolio DI, as their values are larger than $x_{S}$.

\subsection{RORAC diagram}

For a given risk measure, Tasche (1999) justifies the use of the Euler allocation in evaluating performance measured by RORAC across the lines within a firm by establishing that Euler allocation is always RORAC compatible. The RORAC for the entire firm and each line, respectively, 
are defined by

$$
\operatorname{ROR} A C(S)=\frac{-E(S)}{\rho(S)} \quad \text { and } \quad \operatorname{ROR} A C\left(X_{i} \mid \Omega\right)=\frac{-E\left(X_{i}\right)}{\rho\left(X_{i} \mid \Omega\right)}
$$

where $-E(S)$ and $-E\left(X_{i}\right)$ represent the expected profit of the whole firm and line $i$, respectively. As a performance measure the higher RORAC indicates a better performance. However, a problem arises when the allocated capital is negative, because the definition of RORAC (3.6) assigns negative RORACs to the lines with negative allocated capitals despite their superior performance. This inconsistency however is not illogical as Tasche (1999) states: "The case of a positive numerator (profit) and a negative denominator (allocated capital) means that someone gives us a present of a guarantee and even pays for being allowed to do so". To align this situation with the notion of the RORAC (3.6), Tasche (1999) considers two separate cases to explain the concept of the RORAC compatibility stated as follows:

Assuming that the profits of all lines and $\rho(S)$ are positive,

(A) If $-E\left(X_{i}\right) \rho(S)>-\rho\left(X_{i} \mid \Omega\right) E(S)$, then there exists an $\epsilon_{i}>0$ such that

$$
\operatorname{ROR} A C\left(S-h X_{i}\right)<\operatorname{RORAC}(S)<R O R A C\left(S+h X_{i}\right)
$$

for all $0<h<\epsilon_{i}$.

(B) If $-E\left(X_{i}\right) \rho(S)<-\rho\left(X_{i} \mid \Omega\right) E(S)$, then there exists an $\epsilon_{i}>0$ such that

$$
\operatorname{RORAC}\left(S-h X_{i}\right)>\operatorname{RORAC}(S)>\operatorname{ROR} A C\left(S+h X_{i}\right)
$$

for all $0<h<\epsilon_{i}$.

This powerful result enables us to see capital allocation as a valuable risk management metric that can be linked to performance-driven corporate decisions. The statement is somewhat complicated, but this way, problematic cases where the denominators of RORAC in (3.6) are negative can be consistently accounted for. In plain terms, the statement (A) says that, if the $i$ th line performs better than the entire firm, increasing its volume will improve the firm's performance. Likewise, (B) says to downsize the lines performing less than average, where the average means the portfolio RORAC. The rationale of the RORAC compatibility, therefore, tells us to look at RORAC not as a single ratio, but as a set of two separate numbers - the capital (numerator) and the profit (denominator). It is then natural for one to consider the RORAC diagram where each line's performance is marked as

$$
\left(\rho\left(X_{i} \mid \Omega\right),-E\left(X_{i}\right)\right), \quad i=1, \ldots, d,
$$

with the portfolio point $\left(d^{-1} \rho(S),-d^{-1} E(S)\right)$. The benchmark is the straight line connecting the portfolio point and $(0,0)$. Because the slope of the benchmark line represents the firm's RORAC by definition (3.6), it automatically resolves the problematic cases of negative capitals by providing a full capital-profit perspective, as intended by the RORAC compatibility concept. That is, any line marked above (below) the benchmark line, regardless of the sign of its allocated capital, indicates better (poorer) performance and thus is to be expanded (reduced) to improve the portfolio RORAC.

Figure (3.2) shows a RORAC diagram for the working data along with the benchmark. As we define the RORAC as a slope on a plane, its magnitude is easy to perceive, and no complications arise from negative allocated capitals (In fact, lines 3, 5, 8, 9, 10 have negative RORAC values). Lines $1,3,5,8,9,10$ are better performing, as they are above the portfolio RORAC line, and 
thus encouraged to expand, whereas the other lines to be reduced. Another feature of the graph over a simple RORAC comparison is that it accounts for size difference. For instance, the graph differentiates two business lines with the same RORACs but in different dollars by positioning them in different points on the plane.

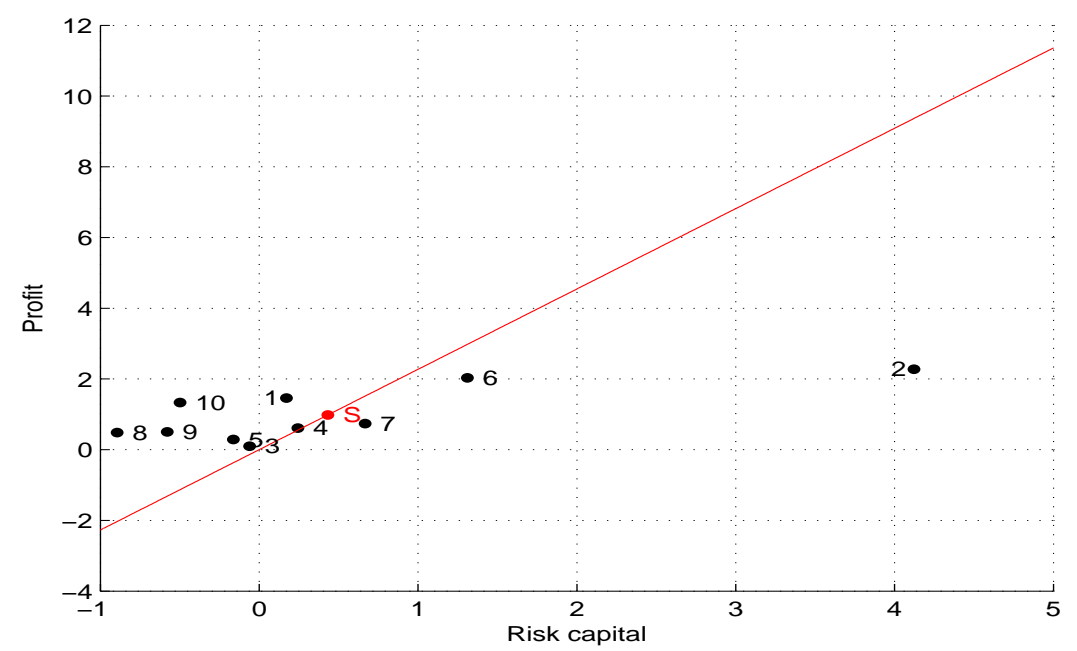

Figure 3.3 RORAC diagram with the benchmark under ES 95\% allocation

If a portfolio arrives at an optimal business mix in terms of the portfolio RORAC, the RORAC values of all business lines will be equal, with all corresponding points on the plane lying along on the portfolio RORAC line, as noted in Tasche (1999). Finding a RORAC-optimal portfolio through iteration however will be computationally intensive especially when the number of lines $d$ is large because the weights can change continuously. We provide a general solution to this later in the article.

\section{Marginal allocation of capital}

The Euler allocation is not the only allocation method. In the literature, an alternative allocation method known as the marginal or incremental risk capital allocation has also been used. The allocated capital under the marginal method is

$$
A C_{i}=\rho(S)-\rho\left(S-X_{i}\right)
$$

This method, while failing to yield the firm's risk capital if added up, as shown in Tasche (1999), has its own rationale and can be found in previous research, including Matten (1996), Chew (2001), Albrecht (2006) and further discussed in Tasche (2004). In this section we establish the relationship of the marginal allocation and the Euler allocation, where the latter is the directional derivative of the former.

The motivation of the marginal allocation is best explained by considering a situation where a firm acquires a new business line. In such a situation, a natural way to determine the line-wise capital would be using the additional increase in the firm's total capital after adding the new line. Note that the Euler allocation, at least conceptually, may not be able to measure the relevant 
risk contribution in such a case because it is based on the directional derivative of the already existing business line. It is sensible therefore to compare the risk capital of the whole portfolio with and without the new line to determine the allocated capital, and that is the idea of the marginal capital allocation.

For our discussion, we start by defining the amount of diversification benefit as

$$
\sum_{i=1}^{d} \rho\left(X_{i}\right)-\rho(S),
$$

which is the difference in the capital under the comonotonic case and the current dependence. One natural candidate of capital allocation is

$$
\sum_{i=1}^{d} \rho\left(X_{i}\right)-\rho(S)=\sum_{i=1}^{d}\left(\rho\left(X_{i}\right)-\rho\left(X_{i} \mid \Omega\right)\right),
$$

so that the diversification benefit assigned to the $i$ th line is

$$
\rho\left(X_{i}\right)-\rho\left(X_{i} \mid \Omega\right),
$$

where $\rho\left(X_{i} \mid \Omega\right)$ is the Euler allocation associated with $\rho$. Note that (4.4) could serve as an alternative measure of diversification; it contains the same information as the DI (3.4), except that the difference is used instead of the ratio.

Now suppose that, without loss of generality, in the firm's current portfolio $\Omega=\left\{X_{1}, \ldots, X_{d}\right\}$, the $i$ th line has recently added from an acquisition. So the firm had only $d-1$ lines prior to the acquisition. Clearly the addition may or may not increase the risk capital for the entire firm. That is, $\rho(S), S=\sum_{k=1}^{d} X_{k}$, could be greater or less than $\rho\left(S-X_{i}\right)$ depending on the relationship of $X_{i}$ with the existing lines $\Omega-\left\{X_{i}\right\}$. However we can show that the diversification amount (4.2) always increases by adding new lines as long as the risk measure is subadditive. That is, the difference of the diversification amount before and after the addition of the $i$ th line is

$$
\begin{gathered}
{\left[\sum_{k=1}^{d} \rho\left(X_{k}\right)-\rho(S)\right]-\left[\sum_{k \neq i} \rho\left(X_{k}\right)-\rho\left(S-X_{i}\right)\right]} \\
=\rho\left(X_{i}\right)+\rho\left(S-X_{i}\right)-\rho(S) \geq 0 .
\end{gathered}
$$

This gives us a way to decompose the risk capital of the newly added line, $\rho\left(X_{i}\right)$, into two sources:

1. The incremental increase in the diversification benefit to the firm by adding line $i$ to the subportfolio $\Omega-\left\{X_{i}\right\}$ :

$$
\operatorname{Ben}\left(X_{i}\right)=\rho\left(X_{i}\right)+\rho\left(S-X_{i}\right)-\rho(S),
$$

which comes from (4.5) and always positive.

2. The incremental change in risk capital (or cost) to the firm by adding line $i$ to the subportfolio $\Omega-\left\{X_{i}\right\}$ :

$$
\operatorname{Cost}\left(X_{i}\right)=\rho(S)-\rho\left(S-X_{i}\right),
$$

which is the marginally allocated capital (4.1), and could be positive or negative. 
In other words, the marginal risk capital added by line $i, \rho\left(X_{i}\right)$, is explained by the sum of the cost towards the newly constructed portfolio and the benefit amount from diversification, so that $\operatorname{Ben}\left(X_{i}\right)+\operatorname{Cost}\left(X_{i}\right)=\rho\left(X_{i}\right)$.

We now show that the seemingly different two allocation methods, the Euler and the marginal allocations, are in fact related. More specifically, the Euler allocation is shown to be the directional derivative of the marginally allocated capital. In addition, we relate two different diversification measures, in (4.4) and (4.6), in a similar fashion.

Lemma 4.1 Let $S(u)=\sum_{i}^{d} u_{i} X_{i}$ where $u=\left(u_{1}, \ldots, u_{d}\right)$ is the exposure (or weight) vector for each line and $X_{i}$ is the net loss for line $i$. Assume the risk measure $f_{\rho}(u)=\rho(S(u))$ is positive homogeneous and continuously differentiable. Under the Euler allocation the directional derivatives of the benefit (4.6) and cost (4.7) are given by, respectively,

$$
\begin{aligned}
\left.\frac{\partial}{\partial u_{i}}\left[\rho\left(S-X_{i}\right)+\rho\left(X_{i}\right)-\rho(S)\right]\right|_{u=1} & =\rho\left(X_{i}\right)-\rho\left(X_{i} \mid \Omega\right), \\
\left.\frac{\partial}{\partial u_{i}}\left[\rho(S)-\rho\left(S-X_{i}\right)\right]\right|_{u=1} & =\rho\left(X_{i} \mid \Omega\right) .
\end{aligned}
$$

Proof: Following the notation of Tasche (2007), we introduce the function $f_{\rho}(u)=\rho(S(u))$, assuming that the probability distribution of random vector $\left(X_{1}, \ldots, X_{d}\right)$ is fixed. Thus $f_{\rho}(u)$ is the risk measure of portfolio at $u$. The current position aggregate loss position is then $S(\mathbf{1})$ with $u_{i}=1$ for all $i$ and its risk measure is $f_{\rho}(\mathbf{1})$. Then, for the cost in (4.7), we have

$$
\operatorname{Cost}\left(u_{i} X_{i}\right)=f_{\rho}(u)-f_{\rho}\left(u_{1}, \ldots, u_{i-1}, 0, u_{i+1}, \ldots, u_{d}\right) .
$$

Therefore

$$
\frac{\partial}{\partial u_{i}} \operatorname{Cost}\left(u_{i} X_{i}\right)=\frac{\partial}{\partial u_{i}} f_{\rho}(u)
$$

which is evaluated at $u=(1, \ldots, 1)$ as

$$
\left.\frac{\partial}{\partial u_{i}} f_{\rho}(u)\right|_{u=\mathbf{1}}=\rho\left(X_{i} \mid \Omega\right)
$$

where the last equation comes from Proposition 2.1 of Tasche (2007). We can similarly prove the benefit case by observing that $\rho\left(u_{i} X_{i}\right)=u_{i} \rho\left(X_{i}\right)$ due to the positive homogeneity of the risk measure. This lemma holds for the ES under some technical conditions discussed in, eg, Lemma 5.6 of Tasche (1999).

Before closing this section, we mention that a result on the marginal allocation by Tasche (2004) holds under more relaxed assumptions than stated in the original paper. For continuously differentiable and sub-additive risk measures that are homogeneous of degree 1, Tasche (2004) shows that the marginal risk contribution (4.7) is always smaller than the corresponding Euler contribution and therefore that the sum of the marginal risk contributions underestimates total risk capital $\rho(S)$. We can prove that the latter result still holds even if the the risk measure is not differentiable. For any $\rho$ that is subadditive and homogeneous of degree 1 ,

$$
\sum_{i=1}^{d} \rho\left(S-X_{i}\right) \geq \rho\left(\sum_{i=1}^{d}\left(S-X_{i}\right)\right)=\rho(d S-S)=(d-1) \rho(S),
$$

which yields

$$
\sum_{i=1}^{d}\left[\rho(S)-\rho\left(S-X_{i}\right)\right] \leq \sum_{i=1}^{d} \rho(S)-(d-1) \rho(S)=\rho(S)
$$




\section{Optimal RORAC portfolio}

While there is a considerable interest in the performance maximization of a firm using the RORAC, see, eg, Crouhy et al. (1999), Tasche (1999, 2004, 2007), Zaik et al. (2005), Saita (2007) and Buch et al. (2011), to name a few, general solution to find the optimal RORAC portfolio has hardly been treated in the literature. For example, as previously mentioned, Tasche (2007) proved that expanding a line with a higher RORAC by a small amount will increase the firm RORAC, but did not discuss how to find it. In this section we show that the optimal RORAC portfolio, when the risk measure is the ES, can be directly obtained by adapting the mean-ES optimization procedure.

\subsection{Formulation}

We start by putting things in the standard financial asset perspective. Consider $d$ risky financial securities with return random variables $R=\left(R_{1}, \ldots, R_{d}\right)^{\prime}$, and denote its mean vector and covariance matrix as $\mu=\left(\mu_{1}, \ldots, \mu_{d}\right)^{\prime}$ and $\Sigma=\left\{\operatorname{Cov}\left(R_{i}, R_{j}\right)\right\}_{i, j=1}^{d}$, respectively. The weight vector $w=\left(w_{1}, \ldots, w_{d}\right)^{\prime}$ represents the weight for each security, which is the decision variable to be optimized. The traditional mean-variance optimization of Markowitz (1959) minimizes the portfolio variance for a given target return. Alternative risk measures other than variance have recently been proposed, as risk measures may not be symmetric. Among these is the mean-ES optimization where one seeks the portfolio minimizing the ES for a given expected return:

$$
\begin{array}{ll}
\text { Minimize } & -E\left[w^{\prime} R \mid w^{\prime} R \leq Q_{1-p}\left(w^{\prime} R\right)\right] \\
\text { Subject to } & E\left(w^{\prime} R\right)=\mu_{c} \text { and } \sum_{1}^{d} w_{i}=1
\end{array}
$$

where $\mu_{c}$ is the target return. The negative sign and quantile level $1-p$ indicates that the variables here are the return (profit) rather than loss. The problem essentially is minimizing the ES under a linear weight constraint. The general solution of the mean-ES portfolio optimization can be found in Rockafellar and Uryasev (2000) and Bertsimas et al. (2004), where the algorithm essentially replaces sorting, usually required for computing quantile-based quantities, with an appropriately chosen linear program involving a small number of constraints and variables. The algorithm works directly with the return sample requiring no assumptions on the return distribution.

The RORAC-optimal portfolio selection procedure resembles the mean-ES optimization above, but there are distinct differences to be addressed carefully. Let us consider a single contract in line $i$ (eg, one auto insurance contract), of which the loss rv and the premium are denoted by $L_{i}$ and $p_{i}$, respectively. The net loss of a contract is then $L_{i}-p_{i}$. If the $i$ th line has $u_{i}$ such contracts in force, we may write the entire firm's loss as

$$
S(u)=\sum_{i=1}^{d} u_{i} \cdot\left(L_{i}-p_{i}\right)
$$

with $u=\left(u_{1}, \ldots, u_{d}\right)$, and the expected profit amount as

$$
E[-S(u)]=-\sum_{i=1}^{d} u_{i} \cdot\left(E\left(L_{i}\right)-p_{i}\right)
$$

We denote the position of the current portfolio as $u^{0}=\left(u_{1}^{0}, \ldots, u_{d}^{0}\right)$. 
Then our RORAC optimization is to find $u$ that solves:

$$
\begin{aligned}
& \text { Maximize } \operatorname{RORAC}(S(u))=\frac{E(-S(u))}{E\left[S(u) \mid S(u)>Q_{p}(S(u))\right]} \\
& \text { Subject to } E(-S(u))=\mu_{c}, \quad \sum_{1}^{d} u_{i} p_{i}=\sum_{1}^{d} u_{i}^{0} p_{i}, \quad u \geq 0 .
\end{aligned}
$$

The budget constraint says the total premium of the new portfolios is kept at the current level, a reasonable place to start, but other choices work as well. We add another constraint $u_{i} \geq 0(i=$ $1, \ldots, d)$, so that negative weights (short selling) are not allowed, as its implications are limited in insurance contexts. The optimal $u^{*}$ will give us a portfolio that has a minimum risk capital for a given profit level, leading to a RORAC-optimal portfolio. As the target profit $\mu_{c}$ changes, we are able to find the globally optimal portfolio. As the net loss amount of a single contract in the $i$ th line is $L_{i}-p_{i}$, we put the rate of return per contract for the insurer as

$$
R_{i}=-\frac{L_{i}-p_{i}}{p_{i}}=-\frac{L_{i}}{p_{i}}+1
$$

This way we treat each line's business as a financial asset with a rate of return $R_{i}$. Using (5.5) we rewrite the firm's loss at position $u$ as

$$
S(u)=\sum_{i=1}^{d} u_{i} \cdot\left(L_{i}-p_{i}\right)=-\sum_{i=1}^{d} u_{i} p_{i} R_{i}
$$

and the expected profit as

$$
E[-S(u)]=\sum_{i=1}^{d} u_{i} p_{i} E\left(R_{i}\right) .
$$

We can then show the following result.

Lemma 5.1 For a fixed premium level, optimizing the RORAC of an insurance portfolio under the ES risk capital as in (5.4) is equivalent to optimizing the mean-ES portfolio as in (5.1).

Proof: Using our notation in this section, if we denote the total premium by $\lambda=\sum_{1}^{d} u_{i} p_{i}$ and $w_{i}=u_{i} p_{i} / \lambda$, then the expected total profit is $\lambda E\left(w^{\prime} R\right)$ and the firm's ES becomes

$$
\begin{aligned}
E\left[S(u) \mid S(u)>Q_{p}(S(u))\right] & =E\left[-\sum_{i=1}^{d} u_{i} p_{i} R_{i} \mid-\sum_{i=1}^{d} u_{i} p_{i} R_{i}>Q_{p}\left(-\sum_{i=1}^{d} u_{i} p_{i} R_{i}\right)\right] \\
& =-E\left[\sum_{i=1}^{d} u_{i} p_{i} R_{i} \mid \sum_{i=1}^{d} u_{i} p_{i} R_{i}<Q_{1-p}\left(\sum_{i=1}^{d} u_{i} p_{i} R_{i}\right)\right] \\
& =-\lambda E\left[\sum_{i=1}^{d} w_{i} R_{i} \mid \sum_{i=1}^{d} w_{i} R_{i}<Q_{1-p}\left(\sum_{i=1}^{d} w_{i} R_{i}\right)\right] \\
& =-\lambda E\left[w^{\prime} R \mid w^{\prime} R<Q_{1-p}\left(w^{\prime} R\right)\right],
\end{aligned}
$$

where $w_{i}$ is the current weight for the $i$ th line with $w_{1}+\ldots+w_{d}=1$. Equipped with these expressions we can rewrite the RORAC optimization (5.4) as:

$$
\begin{array}{ll}
\text { Maximize } & \frac{E\left(w^{\prime} R\right)}{-E\left[w^{\prime} R \mid w^{\prime} R<Q_{1-p}\left(w^{\prime} R\right)\right]} \\
\text { Subject to } & E\left(w^{\prime} R\right)=\mu_{c} / \lambda, \sum_{1}^{d} w_{i}=1, \quad w \geq 0 .
\end{array}
$$


For a constant $\lambda$, the object function's numerator is fixed. Hence the RORAC maximization is equivalent to the mean-ES optimization in (5.1).

The globally optimal portfolio can be found by searching over different profit levels. After the optimal weight $w^{*}=\left(w_{1}^{*}, \ldots, w_{d}^{*}\right)$ has been obtained, the number of contracts to be sold in each line is recovered from $u_{i}=w_{i}^{*} \lambda / p_{i}, i=1, \ldots, d$. Note that $R_{i}$ is a function of premium, so the optimization problem changes as the contract premium varies.

\subsection{Working data result}

We implemented the mean-ES optimization algorithm given in Theorem 3 of Bertsimas et al. (2004) for the working data. Using the equivalence between the mean-ES and the RORAC optimization, we seek the RORAC-optimal portfolio at ES $95 \%$ with the total premium fixed at the current level of 143.797. As Panjer's original data does not provide size information, we simply assume $p_{i}=1$ for each line. Thus, the size of each line $u_{i}$ equals the premium amount.

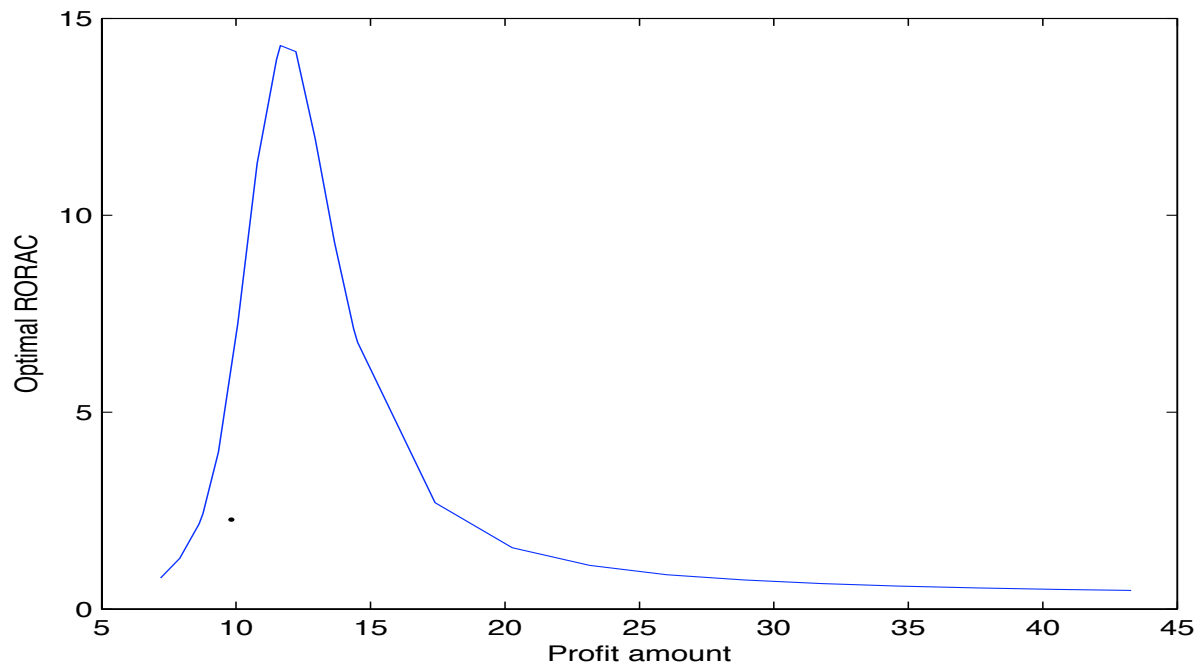

Figure 5.1 Current (dot) vs. RORAC optimial (line) portfolio for the working data at current premium level

$\underline{\text { Table 5.1 Business mix of current vs. RORAC optimal portfolio }}$

\begin{tabular}{cccc}
\hline \hline & Profit & Risk Capital & RORAC \\
\hline Optimal Portfolio & 11.935 & 0.817 & 14.612 \\
Current Portfolio & 9.821 & 4.323 & 2.272 \\
\hline
\end{tabular}

\begin{tabular}{ccccccccccc}
\hline Weight & 1 & 2 & 3 & 4 & 5 & 6 & 7 & 8 & 9 & 10 \\
\hline Optimal & 0.1080 & 0.1722 & 0.0768 & 0.0611 & 0.0088 & 0.1664 & 0.0901 & 0.1354 & 0.1024 & 0.0789 \\
Current & 0.1880 & 0.2788 & 0.0066 & 0.0929 & 0.003 & 0.1807 & 0.1057 & 0.0346 & 0.0342 & 0.0755 \\
\hline Change & -0.0800 & -0.1065 & 0.0702 & -0.0318 & 0.0058 & -0.0143 & -0.0157 & 0.1008 & 0.0682 & 0.0034 \\
\hline
\end{tabular}

The results are reported in Figure (5.1) and Table (5.1). Figure (5.1) shows the highest possible RORAC for different profit levels with the global optimal value at the peak, along with the current position marked as a dot; in this sense, the graph can be thought as the RORAC efficient frontier. The graph lucidly demonstrates the trade-off between profit maximization and diversification 
benefit, and complements the DI and RORAC diagrams. The interpretation of Figure (5.1) is insightful:

- The area under the RORAC curve represents the set of possible portfolio RORAC values with different profit levels. The optimal RORAC curve in the figure can be obtained by connecting the highest RORAC values over a range of different profit levels.

- There is a range in the horizontal axis where the optimal portfolio RORAC increases sharply as one increases the target profit level. This implies that, to a certain level, one can boost the RORAC by both increasing the portfolio profit and lowering the portfolio risk capital thanks to diversification benefit.

- However, once the target profit level gets too high, the optimal RORAC starts to deteriorate and continues to do so. This is because there will be a concentration on more profitable lines to achieve the excessive target profit and, consequently, the risk capital increases with fewer lines to diversify.

- The current position, marked by the dot under the maximum RORAC line, is far from optimal. By shifting its business mix, the firm can reach the peak of the RORAC curve. In fact, from Table (5.1), the optimal business both increase the portfolio profit and decrease the portfolio risk capital compared to the current mix. The resulting improvement in the RORAC is substantial.

Finally we present the RORAC diagram for the optimal portfolio in Figure (5.2), which uses the same scale as Figure (3.3) for an easier comparison. It is seen that each line's RORAC lies around the portfolio benchmark, which confirms our discussion at the end of Section 3. The points do not lie precisely on the benchmark due to the finite sample size of our data. In closing this section, two remarks are in place.

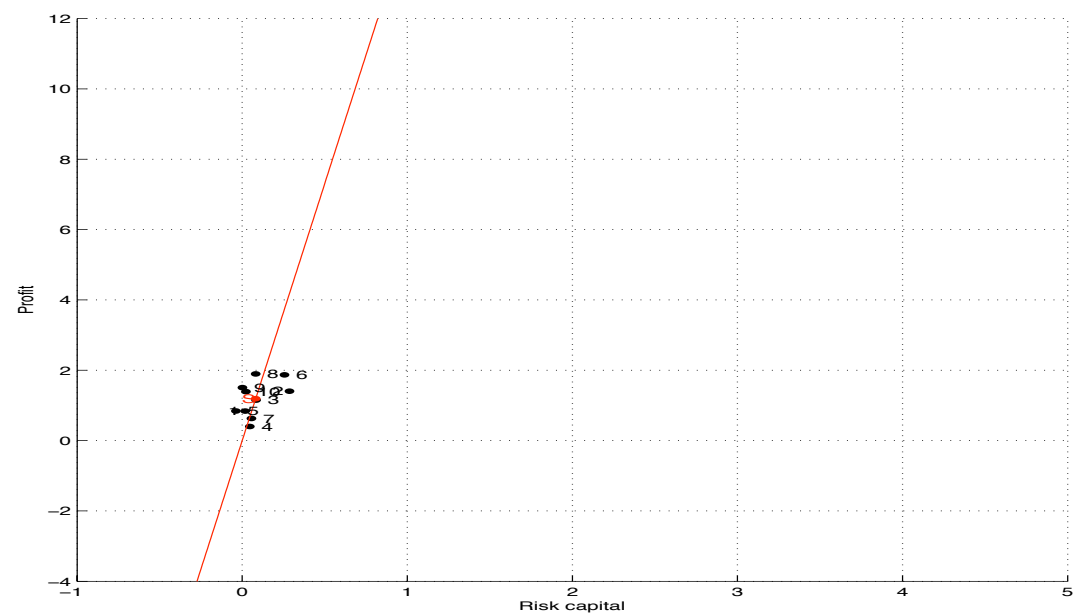

Figure 5.2 SRORAC diagram for the optimal portfolio

Remark 5.1 In practice, the adoption of the RORAC optimization may not be easy as financial firms are constrained by a series of other factors and costs associated with business mix shifts such as marketing channels and administrative costs. In addition, a firm's view on market shares or future strategic vision may be as important as profit-driven business decisions. In this regard, 
the proposed graphs, the DI and RORAC diagrams, can be useful in understanding suboptimal portfolios.

Remark 5.2 Although line-wise RORAC analysis is a good exercise, it should not be directly used to measure the performance of each line's manager. This is because the allocated capital, as we have seen, is a consequence of the dependence among all lines, which is often beyond the control of individual line managers. Therefore one should differentiate the performance of each line from the performance of each line's manager, as also argued in Kim and Hardy (2009). Perhaps the RORAC computed for each line on a stand alone basis may be better for the latter use.

\section{Concluding remarks}

Understanding the contribution and line-wise profitability within a multi-line firm is an important aspect of risk management. In this article we addressed several practical and theoretical issues in capital allocation. First, we proposed several graphs based on the risk metrics of Tasche (1999, 2006, 2007), including the diversification index, line-wise performance and the RORAC efficient frontier. Based on the Euler allocation rule, these visual tools effectively present the interactions among the individual lines. Secondly, on the theoretical side, we proved that the Euler allocation is the directional derivative of the marginal allocation method, an alternative capital allocation rule. Lastly, we used the equivalence between the mean-ES and the RORAC optimization, and showed how to construct the optimal business mix that maximizes the portfolio performance.

\section{References}

Albrecht, P. (2006). Risk based capital allocation. Encyclopedia of Actuarial Sciences, John Wiley \& Sons, New York.

Artzner, P., Delbaen, F., Eber, J. M. and Heath, D. (1999). Coherent measure of risk. Mathematical Finance, 9, 203-228.

BCBS (2006). International convergence of capital measurement and capital standards: A revised framework comprehensive version, Basel Committee on Banking Supervision, Basel.

Bertsimas, D., Lauprete, G. and Samarov, A. (2004). Shortfall as a risk measure: Properties, optimization and applications. Journal of Economic Dynamics and Control, 28, 1353-1381.

Buch, A., Dorfleitner, G. and Wimmer, M. (2011). Risk capital allocation for RORAC optimization. Journal of Banking and Finance, 35, 3001-3009

Chew, D., editor (2001). The new corporate finance: Where theory meets practice, 3rd Ed., McGraw-Hill Companies, New York.

Crouhy, M., Turnbull, S. M. and Wakeman, L. M. (1999). Measuring risk-adjusted performance. Journal of Risk, 2, 5-36.

Denault, M. (2001). Coherent risk allocation of risk capital. Journal of Risk, 4, 1-34.

Dev, A. (2004). Economic capital: A practitioner guide, Risk Books, London.

Dhaene, J., Tsanakas, A., Valdez, E. and Vanduffel, S. (2012). Optimal capital allocation principles. Journal of Risk and Insurance, $\mathbf{7 9}, 1-28$.

Garcia Cespedes, J. C., de Juan Herrero, J., Kreinin, A. and Rosen, D. (2006). A simple multi-factor "factor adjustment" for the treatment of credit capital diversification. Journal of Credit Risk, 2, 57-85.

Good, P. (2000). Permutation tests: A practical guide to resampling methods for testing hypotheses, Springer Verlag, New York.

Gourieroux, C., Laurent, J. and Scaillet, O. (2000). Sensitivity analysis of values at risk. Journal of Empirical Finance, $\mathbf{7}, 225-245$.

Hallerbach, W. (2003). Decomposing portfolio value-at-risk: A general analysis. Journal of Risk, 5, 1-18.

Kalkbrener, M. (2005). An axiomatic approach to capital allocation. Mathematical Finance, 15, 425-437.

Kim, J. H. T. and Hardy, M. R. (2009). A capital allocation based on a solvency exchange option. Insurance: Mathematics and Economics, 44, 357-366. 
Markowitz, H. (1959). Portfolio selection: Efficient diversification of investment, John Wiley \& Sons, New York. Matten, C. (1996). Managing bank capital, John Wiley \& Sons, New York.

McNeil, A. J., Frey, R. and Embrechts, P. (2005). Quantitative risk management, Princeton University Press, New Jersey.

Memmel, C. and Wehn, C. (2006). Supervisor's portfolio: The market price risk of German banks from 2001 to 2004: Analysis and models for risk aggregation. Journal of Banking Regulation, 7, 310-325.

Merton, R. C. and Perold, A. F. (1993). Theory of risk capital in financial firms. Journal of Applied Corporate Finance, 6, 16-32.

Myers, S. C. and Read Jr., J. A. (2001). Capital allocation for insurance companies. Journal of Risk and Insurance, 68, 545-580.

Panjer, H. (2002). Measurement of risk, solvency requirements, and allocation of capital within financial conglomerates, IIPR technical report 01-14, University of Waterloo, Waterloo.

Rockafellar, R. and Uryasev, S. (2000). Optimization of conditional value-at-risk. Journal of Risk, 2, 21-42.

Saita, F. (2007). Value at risk and bank capital management, Academic Press, London.

Sherris, M. (2006). Solvency, capital allocation and fair rate of return in insurance. Journal of Risk and Insurance, 73, $71-96$

Sherris, M. and van der Hoek, J. (2006). Capital allocation in insurance: economic capital and the allocation of the default option value. North American Acutarial Journal, 10, 39-61

Shim, J. and Hwang, C (2013). Expected shortfall estimation using kernel machines. Journal of the Korean Data ES Information Science Society, 24, 625-636.

Skoglund, J. and Chen, W. (2009). Risk contributions, information and reverse stress testing. The Journal of Risk Model Validation, 3, 61-77.

Tasche, D. (1999). Risk contributions and performance measurement. Working paper, Zentrum Marhematic (SCA), TU Munchen, Germany.

Tasche, D. (2004). Economic capital: A practitioner's guide. In Allocating Portfolio Economic Capital to Subportfolios, edited by A. Dev, Risk Books, London, 275-302.

Tasche, D. (2006). Measuring sectoral diversification in an asymptotic multifactor framework. Journal of Credit Risk, 2, 33-55.

Tasche, D. (2007). Euler allocation: Theory and practice. Arxiv preprint arXiv:0708.2542.

Zaik, E., Walter, J., Retting, G. and James, C. (2005). RAROC at bank of america: From theory to practice. Journal of Applied Corporate Finance, 9, 83-93.

Zanjani, G. (2002). Pricing and capital allocation in catastrophe insurance. Journal of Financial Economics, 65, 283-305. 